du nourrisson à l'aide de normes portant sur les avantages de l'allaitement maternel exclusif.[6]

La littérature scientifique suggère déjà la présence d'une tension entre le discours de l'allaitement maternel exclusif et les approches tolérant une alternative à ce type d'alimentation du nourrisson favorisant d'autres modes pour le nourrisson. Cette tension a pour effet de soulever plusieurs enjeux éthiques au sein même de la pratique infirmière. Dans un premier temps, le rôle de promotion de l'allaitement maternel exclusif chez le personnel infirmier[6] présente un risque de dissonance lorsqu'il se heurte à une approche de soins qui se veut centrée sur la mère, si cette dernière rencontre des difficultés d'allaitement ou ne choisit pas l'allaitement maternel exclusif pour son nourrisson.[8] Subséquemment, l'infirmière risque aussi de contraindre la relation de soin entretenue avec la patiente[9] ayant pour effet d'entraver la qualité de l'accompagnement lui étant offert.[10,11,12,13]

Plusieurs études qualitatives récemment menées sur le sujet montrent l'existence d'un sentiment de culpabilité chez les mères ne choisissant pas l'allaitement maternel exclusif[14,15,16,17,18,19,20] voire même, un sentiment d'échec [21,19]. L'étude exploratoire de Hvatum et Glavin[12] suggère que ces sentiments de culpabilité, de honte et d'échec s'expliquent par le désir des mères à se conformer aux normes favorables à l'allaitement maternel exclusif. De plus, la transmission d'informations éclairées sur le choix du mode d'alimentation du nourrisson est un enjeu éthique soulevé dans la littérature.[22] En ce sens, l'accessibilité de l'information et la modification de la disponibilité des informations sont des stratégies de santé publique utilisées par le gouvernement visant à favoriser l'adoption de comportements de santé sains.[23] En l'occurrence, cette stratégie génère un certain contrôle sur l'acquisition, I'utilisation, la rétention et la transmission d'informations véhiculées au grand public.[23] Conséquemment, l'étude de Fallon et ses collaborateurs[10] stipule que seulement $36 \%$ des mères de leur échantillon (601 mères) qui avaient choisi le biberon se sentaient soutenues par les professionnels de la santé. En d'autres termes, cette tension entre les standards de pratiques favorables à l'allaitement soulève des enjeux éthiques pour l'infirmière quant à la transmission d'informations éclairées sur les choix d'alimentation du nourrisson[22] additionné à l'accompagnement des femmes à l'égard leurs prises de décision alternative à l'allaitement exclusif.[20]

Cette recherche qualitative a pour but de comprendre les tensions éthiques et cliniques à l'œuvre lorsque les pratiques de soin favorables à l'allaitement maternel exclusif se heurtent aux choix alternatifs des mères à cet égard. Elle s'appuie sur la théorie des soins transculturels de Madeleine Leininger[24] et l'approche méthodologique s'inspire de la démarche d'Adele Clarke[25] afin d'analyser le discours des mères qui choisissent des alternatives à l'allaitement maternel exclusif. L'analyse situationnelle est une approche itérative qui tient compte de la perspective principale de la personne, acteur social central à l'étude, afin d'observer l'ensemble de l'univers social dans lequel la personne évolue.[25] Cette recherche vise à répondre à la question suivante : comment le choix d'une alternative à l'allaitement maternel exclusif est-il discursivement construit par les mères lorsqu'il se confronte aux pratiques institutionnelles et aux normes sociales? L'objectif principal est d'explorer comment s'articule le choix du mode d'alimentation du nourrisson par les mères qui optent pour une alternative à l'allaitement maternel exclusif. L'objectif secondaire vise à décrire l'influence des normes associées à la performance parentale et maternelle sur la prise de décision des mères à l'égard du mode d'alimentation du nourrisson. L'objectif tertiaire est de décrire les effets d'un tel choix sur l'expérience générale de la maternité telle qu'elle est mise en discours par les mères.

\section{Cadre théorique}

Le cadre de référence infirmier utilisé pour cette analyse est celui du soin transculturel de Leininger.[24] L'utilisation de ce cadre de référence vise principalement à comprendre les enjeux socioculturels des pratiques d'allaitement à travers le processus de maintien, de négociation et de restructuration du soin dans une culture de soin ou l'allaitement est présenté telle une norme. Dans sa pratique, l'infirmière peut préserver les soins culturellement congruents qui sont favorables à l'allaitement pour les mères qui souhaitent allaiter, accommoder les soins pour les mères qui formulent un choix partagé ou expriment des contraintes, ou finalement restructurer les soins pour les mères qui choisissent de ne pas allaiter. Dans cette visée, les participantes qui font des choix alternatifs à l'allaitement maternel exclusif évoquent une sous-culture. Les discours des participantes ont permis d'observer le pluralisme normatif de la culture de soin favorable à l'allaitement au sein duquel différentes normes se rejoignent ou se heurtent avec une force variable. La théorie de Leininger apporte une compréhension socioculturelle des pratiques d'allaitement des mères lorsque ces dernières s'éloignent des normes caractérisant les pratiques de soin favorables à l'allaitement. À travers les nombreuses initiatives visant à promouvoir l'allaitement, dont I'IAB, l'infirmière doit assister à certaines formations portant sur l'allaitement, offrir un apprentissage de qualité portant sur l'allaitement, adhérer 
au contrôle des publicités à l'égard des formules lactées, favoriser la mise en place des dix conditions pour un succès de l'allaitement, etc.[5] Par conséquent, ces éléments évoquent des représentations non matérielles de la culture favorable à l'allaitement, éléments convoités de concert avec Clarke.[25]

\section{Approche méthodologique qualitative inspirée de la théorisation ancrée}

L'approche de type qualitative convoitée pour cette recherche est fondée sur l'analyse situationnelle du discours de mères qui ont choisi un mode alternatif à l'allaitement maternel exclusif. Cette approche développée par Adèle Clarke met l'emphase sur la perspective de personne comme élément central de l'analyse afin d'observer l'ensemble de l'univers social dans lequel elle évolue.[25] Cette analyse tient compte des discours, des silences et de l'influence du non humain qui évoque, entre autres, les produits culturels, la technologie, les animaux, les médias, et tant d'autres objets ayant une influence sur les représentations des personnes sur le monde vécu.[25] L'analyse des données s'opère à partir de trois styles de cartographies principales que Clarke[25] présente comme étant les cartes situationnelle, positionnelle et du monde social/arène. Ces cartographies visent à décrire l'ensemble des éléments inhérents à la situation d'étude (exemple la figure 1) ainsi que les liens qui s'opèrent entre ceux-ci, les mondes sociaux ainsi que leurs chevauchements et les prises de position ou les absences de prise de position.[25] Le tout s'opère à partir d'une analyse du discours inspirée de la théorie foucaldienne, une importance marquée pour le non-humain et une considération importance pour les silences.[25] L'analyse situationnelle s'inspire des théories féministes duquel découle des concepts sensibilisant à l'objet d'étude dont la maternité intensive[17] ou le sacrifice féminin de la maternité.[26]

\section{Collecte des données}

Pour participer à cette étude, les participantes devaient avoir choisi une alternative à l'allaitement maternel exclusif et avoir accouché au Québec après 2008, date à laquelle le Ministère de la Santé et des Services sociaux s'est octroyé le mandat de certification des établissements IAB.[5] L'année 2008 représente ainsi une date symbolique en ce qui concerne la culture de pratique favorable à l'allaitement maternel au Québec, malgré que I'on constate l'existence de telles pratiques bien avant cette date. Le recrutement s'est effectué durant l'été 2018 avec l'aide d'une affiche virtuelle présentée

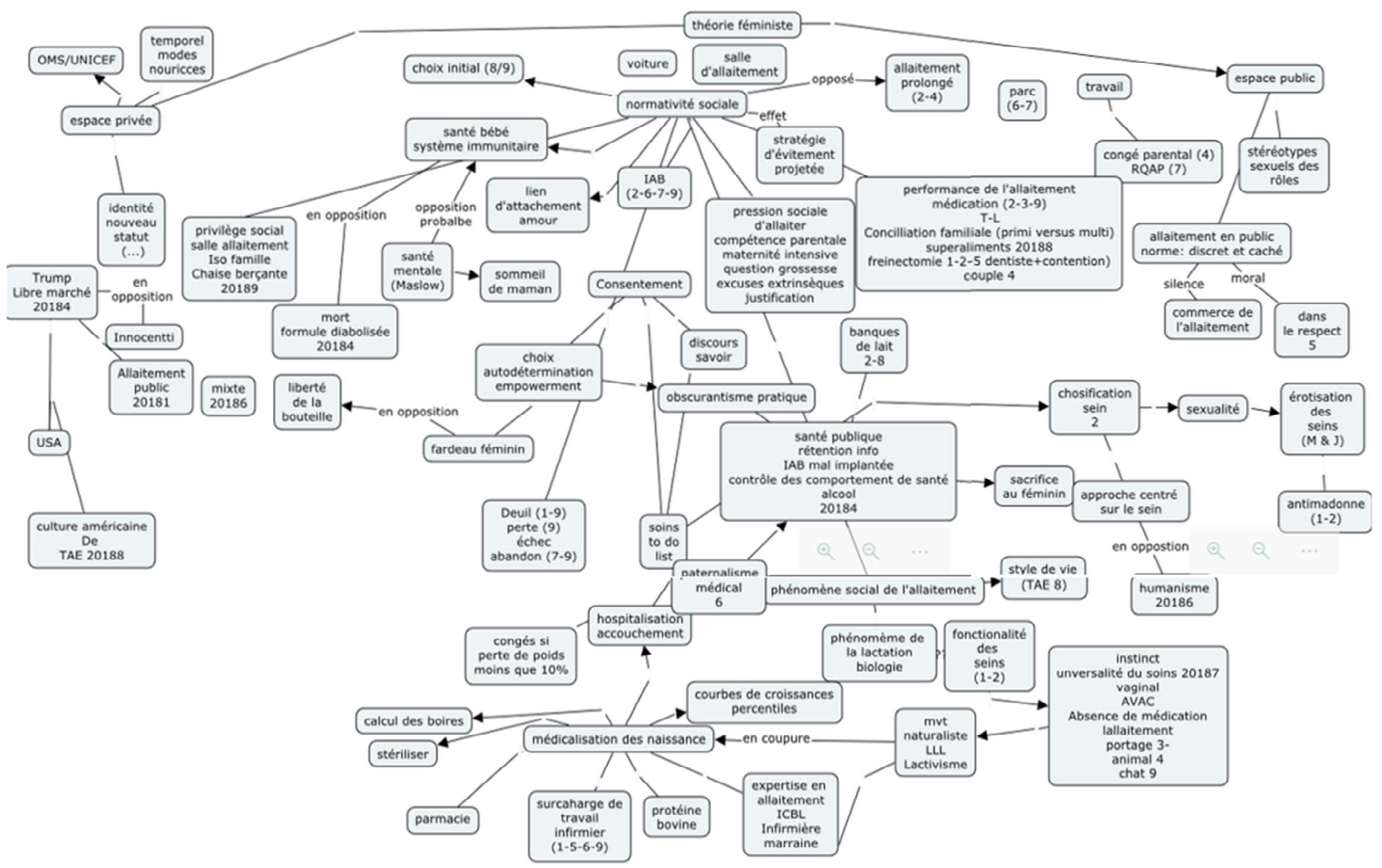


sur les réseaux sociaux. Ce type de recrutement passif visait une participation libre et volontaire. À cet effet, un formulaire de consentement a été signé pour chacune des participantes et leur anonymat a été respecté au cours de cette recherche qui a été approuvée par le comité d'éthique de I'Université du Québec en Outaouais (UQO).

Des questionnaires sociodémographiques (tableau 1) et des entrevues semi-dirigées d'une durée approximative d'une heure trente ont été menés auprès des participantes de cette étude. Les entrevues ont été enregistrées sous forme audio et ont ensuite fait l'objet d'une transcription des verbatim. La confidentialité a été assurée par la codification des prénoms et noms des participantes. L'utilisation de réflexions personnelles d'introspection, la rédaction d'un journal de bord et l'apport de rapport post-entrevue sont des outils qui ont été utilisés afin de maximiser la réflexivité entourant cette problématique. Les résultats ont été analysés simultanément à la collecte des données sous forme de cartographie et d'analyse thématiques extraites à même les transcriptions du verbatim des entrevues. Au total, 14 expériences d'accouchement ont été partagées par les neuf participantes âgées de 26 à 40 ans, dont quatre primipares. Finalement, quatre participantes ont décrit l'alternative à l'allaitement maternel exclusif comme étant de la formule lactée exclusivement tandis que pour cinq des participantes, elle consistait en un mélange entre du lait maternel, obtenu par mise au sein ou extraction, et de la formule lactée.

\section{Résultats}

Les résultats de cette recherche ont permis d'extraire trois principaux thèmes, qui sont : 1) le maintien des normes culturelles de l'allaitement maternel exclusif; 2) l'ajustement et la négociation des pratiques d'allaitement; et, 3) l'allaitement maternel exclusif comme enjeu de performance maternelle. Ce présent article vise à présenter les deux premiers thèmes obtenus avec l'aide de l'analyse des verbatim et des cartographies.

\section{1. le maintien des normes culturelles de l'allaitement maternel exclusif: un enjeu de négociation du soin.}

Ce premier thème présente principalement la construction du choix alternatif à l'allaitement maternel exclusif. Cette construction du choix évoque le choix initial, les contraintes décisionnelles, ainsi que le processus parfois complexe et difficile d'éclaircissement du choix.

Choix initial. D'abord, le choix du monde d'alimentation du nourrisson en période de préconception s'exprimait, pour la plupart, par un désir initial d'allaiter : " J'ai fait beaucoup de lectures sur le sujet et moi je m'étais toujours dit que j'allais allaiter mes enfants " (P8). Les deux principaux motifs évoqués étaient l'apport de l'allaitement sur la santé du nourrisson et sur le lien d'attachement. Pour la participante suivante, la santé du nourrisson était présentée de façon dichotomique à la maladie et l'allaitement diminuerait ainsi les risques que l'enfant soit malade : "Au niveau de la santé de l'enfant,

\begin{tabular}{|c|c|}
\hline Données sociodémographiques & Nombre / \% \\
\hline \begin{tabular}{|ll} 
Âge en 2018 & \\
& - Entre 26 et 30 ans \\
& - Entre 31 et 35 ans \\
& - Entre 36 et 40 ans
\end{tabular} & $\begin{array}{ll}4 & 44,0 \% \\
1 & 11,0 \% \\
4 & 44,0 \%\end{array}$ \\
\hline \begin{tabular}{|} 
Dernier diplôme scolaire obtenu \\
-Secondaire \\
-Collégial \\
-Baccalauréat \\
-Maîtrise
\end{tabular} & $\begin{array}{ll}1 & 11,0 \% \\
2 & 22,0 \% \\
4 & 44,0 \% \\
2 & 22,0 \%\end{array}$ \\
\hline \begin{tabular}{|r} 
Statut matrimonial \\
-Conjoint de fait \\
-Mariée
\end{tabular} & $\begin{array}{ll}7 & 78,0 \% \\
2 & 22,0 \%\end{array}$ \\
\hline $\begin{array}{r}\text { Durée du congé parental } \\
\text {-De six mois à un an } \\
\text {-Plus d'un an }\end{array}$ & $\begin{array}{ll}7 & 78,0 \% \\
2 & 22,0 \%\end{array}$ \\
\hline $\begin{array}{r}\text {-Un enfant } \\
\text {-Deux enfants }\end{array}$ & $\begin{array}{ll}4 & 44,0 \% \\
5 & 56,0 \%\end{array}$ \\
\hline
\end{tabular}


c'était que ça (l'allaitement) favorise les anticorps et que les enfants risquaient moins d'être malades " (P7).

Contraintes décisionnelles. Dans la littérature scientifique, comme dans l'espace public, il est possible de retrouver une association entre l'image de la bonne mère et l'allaitement.[14,15,17,27,9,28] À cet effet, les mères qui veulent répondre aux injonctions favorables de la maternité détiendraient à première vue un espace décisionnel amoindri.[29] À cet effet, les participantes de cette étude remettaient en question le caractère libre du choix qui était fait à l'égard du mode d'alimentation du nourrisson. En conservant leur liberté de choisir, elles devaient malgré tout négocier les contraintes symboliques associées aux injonctions sociales de la bonne maternité. La déception relative au choix du biberon est présentée par cette participante, qui dénote une contrainte bien réelle contribuant à nuancer la décision exercée: "On dirait que si tu te gardes une porte ouverte pour l'allaitement, le biberon est comme en dépit. Mais jusqu'où tu te rends, dans le fond ? "(P9). Cette perception de choix imposé semble aussi s'expliquer par la présentation de la supériorité du lait maternel retrouvé dans le discours de plusieurs participantes. Tel que : "La formule c'est moins bon. Le fer dans l'allaitement, c'est mieux " (P7). Dans cette visée, Thomson et al.[30] stipulent que le fait de donner le biberon entraine le sentiment de ne pas agir dans le meilleur intérêt de son enfant naissant afin de lui assurer un bon départ. Cette notion de bon départ se retrouve au sein même de l'IAB, qui dépeint l'allaitement comme une pratique assurant " à chaque enfant, le meilleur départ possible dans la vie ".[5] Cette présentation évoque la conception de la maternité intensive qui présente le rôle de la mère telle une dévotion complète du plein potentiel de l'enfant.[17] Cette présentation de la maternité entrainerait une forme de performance qui n'est pas toujours compatible avec les autres sphères de la vie, dont la vie familiale.[28]

La représentation sociale du lait maternel comme étant supérieur aux autres modes d'alimentation s'illustre bien dans les propos de cette participante : "On avait justement lu beaucoup sur le lait maternel, c'est la meilleure des options" (P4).

Éclaircissement du choix. Dans le discours de toutes les participantes, les bénéfices de l'allaitement étaient présents. À ce sujet, plusieurs éléments étaient évoqués dont la simplicité : "Moi je trouvais ça (l'allaitement) simple et efficace " (P6) et l'aspect pratique de l'allaitement: "C'est pratique, tu as tout le temps du lait à la portée de main, à la bonne température "(P5). En contrepartie, les désavantages de l'allaitement se retrouvaient peu dans le discours des participantes. Pourtant, les participantes ont toutes nommé l'impact négatif que pouvait avoir l'allaitement sur la santé mentale des mères. Pour cette participante, les impacts se retrouvaient tant sur le plan physique que mental : "J'aurais aimé que ça (l'allaitement) fonctionne, mais j'ai fait le choix. Entre être épuisée pour pouvoir donner du lait (maternel), choisir d'être une maman plus en forme et plus patiente" (P8). Toutefois, la fatigue ou encore le temps que nécessite l'allaitement n'étaient pas énoncés directement sous forme de désavantage de l'allaitement. Certains avantages de la formule lactée donnée par le biberon ont été soulevés dont un retour au travail plus rapide, le partage des boires avec l'autre parent et une plus grande liberté d'action. D'ailleurs, l'étude de Nihlén Fahlquist[19] présentait des propos de participantes qui associaient l'allaitement au manque de liberté d'action. Selon Badinter, l'allaitement à la demande du bébé brimerait le droit à la femme d'avoir du temps pour elle-même et le biberon proposerait un compromis libérant la femme de son rôle de mère afin de concilier ses besoins individuels à ceux du bébé.[26] Les propos de cette participante évoquent bien cette association entre la liberté d'action et l'utilisation du biberon :

Si je voulais sortir, j'avais quand même la liberté de pouvoir laisser (le bébé) à papa, ou mes parents, ou peu importe. Sans me sentir mal ou (me) dire (qu') il faut que je sois revenue dans une heure parce qu'il a soif (P2).

Finalement, les désavantages de la formule lactée donnée par le biberon relaté par plusieurs participantes regroupaient principalement : la logistique de la préparation, les coûts et le manque d'information en général. Plusieurs études soulèvent le manque d'information générale sur l'utilisation des formules lactées.[10,12,31,18,32] Certaines mères vivraient même une insatisfaction lorsqu'elles cherchent à obtenir de l'information portant sur les formules lactées auprès du personnel médical.[13] L'extrait suivant illustre cette insatisfaction :

Tu n'as pas de soutien non plus. Moi j'ai une amie pour qui l'allaitement n'avait pas fonctionné. Donc je me suis beaucoup référé à elle, mais l'infirmière du CLSC ne m'avait pas guidé sur comment augmenter les quantités de lait dans le biberon (...) même le pédiatre ne savait pas trop quoi me dire. Tu manques un peu de ressources (P9).

Selon Gostin[23], la santé publique détiendrait un certain contrôle sur les informations transmises et les comportements à adopter (ou éviter) afin d'optimiser la santé. La formule lactée s'inscrit culturellement tel un choix qui induit un certain niveau de risque pour la santé des bébés.[17,29] L'association symbolique de la formule lactée aux risques pour 
la santé peut s'expliquer par l'absence de présentation des désavantages de l'allaitement, voir même des avantages du biberon dans les discours des participantes. Dans cette visée, la présentation des bienfaits de l'allaitement et des risques de la formule lactée évoquerait une rationalité qu'opère la santé publique dans la rétention et la distribution d'informations au regard des comportements favorables (allaitement) ou risqués (formule lactée) pour la santé.[30,33]

\section{Ajustement des pratiques d'allaitement : entre restructuration et persuasion}

Le second thème émergeant des résultats présente une tension entre deux paradigmes actuels qui coexistent dans les soins offerts aux parturientes en milieu hospitalier. Cette cohabitation de paradigmes, soit la médicalisation des naissances et la culture de soins favorable à l'allaitement, crée des confrontations dont la force est variable et dont les effets sur le vécu des mères sont palpables lorsque leurs discours sont étudiés. Le premier paradigme de la médicalisation des naissances offre, à titre d'exemple, un contrôle de paramètres, dont les ingestions du bébé qui aurait historiquement été facilité avec l'implantation de la formule lactée.[34] Toutefois, la huitième des dix conditions pour un succès de l'allaitement maternel, qui constitue aussi un critère d'accréditation des établissements certifiés par I'IAB, prévoit d'encourager l'allaitement à la demande du bébé.[5] L'allaitement à la demande serait ainsi en rupture avec le calcul de la quantité de lait ingéré par le bébé et cadré selon un horaire. Tout comme Rivard[35] le propose, l'extrait suivant illustre la coexistence de ces deux paradigmes qui cohabitent au sein des guides informatifs remis aux parents : "Mes amies qui allaitent se font demander la quantité de lait que bébé boit (...) C'est écrit dans le Mieux-Vivre, un bébé de tant de mois est (censé) boire tant de quantité. Mes amies qui allaitement me le disent : mais je ne sais pas qu'elle quantité (il boit). " (P2). Ces discours contradictoires ont pour effet de créer de l'incertitude pour certaines mères, qui a été exprimée de la manière suivante par une participante : "Le discours n'est pas toujours uniforme. Pour certains parents, c'est difficile de s'y retrouver et ça peut même créer du stress, parce que tu ne sais plus à qui te fier " (P5).

Influence des pratiques de soins médicalisées sur le vécu des mères. La plupart des participantes ont fait référence à l'existence de pratiques spécialisées en allaitement, ce qui témoigne de la mise en place d'un discours d'expertise professionnelle en la matière. D'ailleurs, le développement d'une expertise en allaitement serait une des visées de la médicalisation de la nutrition du nourrisson.[34] Le terme
" spécialiste " a été évoqué à plusieurs reprises: "On a vu une pro en allaitement (...) la spécialiste en allaitement " (P1). [Et] " Une infirmière en lactation, elle était spécialisée làdedans " (P8). De cette expertise pouvait découler un discours d'autorité professionnelle dont les injonctions centrées sur la responsabilisation maternelle ont produit un sentiment de culpabilité chez cette participante :

II (le pédiatre) regarde sur la courbe (de croissance), puis il me chicane : Oui, bien là ton enfant, il ne prend pas assez de poids. Tu n'allaites pas assez. Bien là, comment ça, je n'allaite pas assez ? En même temps, je me disais : comment on fait pour allaiter plus? (P4)

Le discours d'expertise sur l'allaitement auquel adhèrent les professionnels de la santé était, pour une autre participante, un enjeu de pouvoir qui positionne la mère dans une situation de soumission à l'autorité. Les mots " influençable ", " ne connais pas " et " embobiner " font l'éloge d'un manque de contrôle perçu par celle-ci :

Parce que si tu es moindrement influençable, et que tu ne connais pas ça, et que, disons, soit quelqu'un qui n'a pas d'éducation, soit quelqu'un qui ne connait pas du tout le milieu médical, bien tu te fais embobiner làdedans. Facilement (P6).

Différents enjeux de pouvoir entre l'expertise médicale et l'autonomie des mères émergeaient des discours des participantes. Ainsi, l'absence de contrôle sur la situation et l'emprise du cadre médical était bien présentée par cette participante, qui attribue la fin de l'hospitalisation aux critères unilatéralement définis par l'autorité médicale. L'allaitement représentait un enjeu pour l'obtention du congé de I'hôpital pour la participante suivante, puisque la prise de poids du bébé était insatisfaisante selon les paramètres médicaux établis : " II (bébé) faut qu'il mange. II a perdu presque deux livres. Vous ne me laissez pas sortir de l'hôpital s'il ne prend pas de poids, mais vous refusez qu'il prenne autre chose que le sein " (P6). Cet extrait présente la cohabitation entre les approches de médicalisation de soin et celles plus favorables à l'allaitement exclusif de laquelle découle également un processus de culpabilisation systématique des mères qui se retrouvent au cœur de ces tensions.

Pour représenter ces enjeux de pouvoir, la forme symbolique a été utilisée par une participante: " Elle (l'infirmière) est arrivée avec ses gros sabots dans notre chambre pour nous expliquer (la mise au sein) " (P1). Cet extrait représente l'infirmière par sa rudesse tout en la positionnant telle une figure d'autorité qui explique la mise au sein aux femmes devenues mères. Pourtant, une approche rude et directive des professionnels de la santé serait associée à un support négatif et défavorable au développement de la confiance en soi chez les mères.[36] 
Le langage non verbal de l'infirmière responsable de ses soins a aussi été soulevé par cette participante: "Je revois l'infirmière dans la porte de la chambre qui dit : Vous choisissez de donner le biberon. Avec (...), les mains sur les hanches. Je dis oui. Bien, elle dit, on n'en a pas ici " (P9). Dans cet extrait, le choix des mots rend explicite l'absence de disponibilité quant au choix alternatif à l'allaitement additionné aux enjeux de pouvoirs exprimés par différentes stratégies informelles et persuasives telles que l'attitude et l'utilisation d'une posture d'autorité. La transgression de certaines limites personnelles de la part des infirmières envers les participantes a aussi été soulevée. Certaines interventions auxquelles ont été soumises les participantes ont été vécues de façon intrusive et irrespectueuse de leur espace privé. Une participante présentait cette intrusion par rapport à la notion de soi, notamment parce que son consentement n'a pas été recherché avant d'effectuer un soin sur elle-même et sur son enfant: "Puis une infirmière, qui te touche le sein, prend ton bébé comme ça, c'est, à moi. C'est mon enfant. Ne prends pas mes choses "(P7). Pour cette autre participante, c'est le sentiment de dépossession de son corps qui exprimait cette intrusion, soulevant par le fait même une forme d'objectification du sein, ici construit comme un objet détaché du corps féminin dont la fonction unique serait de nourrir le bébé :

Un moment donné, à l'hôpital, je me disais, ok, mon corps ne m'appartenait plus. La première fois, l'infirmière avec le tire-lait elle m'a ouvert la jaquette. Prit le sein. Ramasser le tire-lait. (...) Moi j'avais mal, je voulais juste m'en aller chez moi, c'était juste ça, donc pogne les, mes seins, à la limite, fais juste nourrir (P9).

La perméabilité des frontières entre les espaces privé et public dans un contexte d'allaitement est également présentée par ce sentiment d'intrusion dans la vie familiale des participantes. Le sein de la participante, généralement confiné à la sphère privée, serait ainsi politisé et investit des injonctions sociales associées à la maternité et à l'allaitement. Cette déconstruction du corps rappelle le paradigme de la médicalisation des naissances qui vise un contrôle de paramètres spécifiques, la lactation, dissociée de l'allaitement comme pratique corporelle, culturelle, sociale et comme un rituel familial.[34] Le pouvoir, le savoir et l'autorité du personnel médical représentent ici la sphère publique, mais également l'autorité professionnelle investit des discours de la santé publique portant sur l'allaitement maternel.

Effet de la culture du soin favorable à l'allaitement sur le vécu des mères. Différentes représentations de la culture de soin favorable à l'allaitement se retrouvaient dans les discours des mères. Certaines nommaient, par exemple, l'autorité perçue des instances gouvernementales: "Santé Canada préconise l'allaitement par rapport à la formule [lactée] " (P1). Pour d'autres, l'idéologie favorable à l'allaitement présente au sein de l'environnement culturel était soulevée. Cette participante fait directement référence à l'IAB :

Je trouvais dommage aussi que les hôpitaux ont la bannière, amis des bébés. C'est, amis des bébés, mais, selon l'allaitement. C'est amis des bébés pour l'allaitement, mais pour moi amis des bébés c'est de s'adapter aux besoins du bébé et de la maman (...) je trouve que ça ne reflète pas la réalité. Bien, ça reflète la réalité d'un environnement où l'on prône que l'allaitement exclusif. Mais ça ne reflète pas la réalité d'une maman qui n'est pas capable d'allaiter, ou que ça ne fonctionne pas avec son bébé, ou qui veut juste autre chose. Ça ne reflète pas cette réalité-là. Mais ça reflète la réalité des hôpitaux qui prônent l'allaitement exclusif (P2).

\begin{tabular}{|c|c|}
\hline $\begin{array}{l}\text { Données socio- } \\
\text { démographiques }\end{array}$ & Logistique \\
\hline Cuillère: & $\begin{array}{l}\text { Puis une heure après avoir allaité, tu dois tirer ton lait. Puis ce que tu aurais récolté, faut le donner à la } \\
\text { cuillère. (...) Une cuillère en plastique. De cafétéria. Un peu tranchante sur le bord. (...) Voyons donc. C'est } \\
\text { un bébé. Je n'étais pas prête à ça. (...) Voir que je vais lui donner le peu (de lait recueilli, puis il (le bébé) se } \\
\text { débattait. (...) De toute façon, j'en avais tellement pas, c'est ça qui est ridicule (P9). }\end{array}$ \\
\hline $\begin{array}{l}\text { Contenant à médi- } \\
\text { caments : }\end{array}$ & $\begin{array}{l}\text { Ils ont essayé ça, le gobelet, la petite affaire de médicaments. C'est ce qui a le mieux marché. Puis c'est } \\
\text { vraiment un hasard, c'est l'infirmière qui a dit c'est assez. Mais elle ne voulait pas essayer le biberon (P6). }\end{array}$ \\
\hline Seringue: & $\begin{array}{l}\text { Je tirais le colostrum, en fait je prenais le colostrum avec des massages, dans un petit cup (récipient) qui } \\
\text { donnait puis, après cela, il (le personnel hospitalier) le mettait dans une seringue, et puis on lui donnait } \\
\text { avec le doigt (P2). }\end{array}$ \\
\hline Téterelle: & $\begin{array}{l}\text { Ils (le personnel hospitalier) m'ont dit, bon prends une téterelle. Mais une téterelle, c'est comme une tétine } \\
\text { en silicone, que tu mets, pour qu'il y ait un bout qui colle. Ça ne marchait pas, le petit (bébé) s'enrageait } \\
\text { parce que ça arrachait. Ça ne tenait pas. On a dit, ok, tirer le lait ça ne fonctionnait pas non plus. Et puis, on } \\
\text { s'est mis les deux (couple) à pleurer (P7). }\end{array}$ \\
\hline
\end{tabular}


Un des critères d'accréditation $I A B$ en milieu hospitalier est le taux d'allaitement fixé à 75\%.[5] À cet effet, une des participantes remettait en question l'existence de ce taux à atteindre: "Un moment donné, je me suis demandé s'il n'avait pas une cote sur le nombre de mères qui allaitent " (P9). Les résultats de l'étude de Hunt et Thomson[11] suggèrent que les mères ont parfois l'impression que le personnel médical utilise une approche fonctionnelle et théorique de l'allaitement dans le but de répondre à certaines directives. Pour cette participante, la culture de soins favorable à l'allaitement a eu un effet de contrôle de l'information et une absence conséquente de support à sa décision :

Elle (l'infirmière) est venue, nous voir et nous a dit : on le savait, que tu ne pouvais pas allaiter, mais on ne pouvait pas te le dire. On savait que ça ne marcherait pas. Mais on est tellement pro-allaitement. (P7)

Lakshman et ses collaborateurs[21] proposent que certains professionnels de la santé n'offrent aucun support portant sur le biberon en période postnatale, ce qui fait écho aux propos de cette participante: "Dès que cette personne-là a pris sa décision (référant aux mères), qu'elle (l'infirmière) soit en accord ou pas avec toi, il faut que tu la respectes. Ça devient du respect de l'humain "(P6). Dans un autre ordre d'idée, plusieurs instruments visant à maintenir l'allaitement maternel exclusif se retrouvaient dans la culture de soins favorable à l'allaitement. L'utilisation d'une cuillère, d'un contenant à médicament, d'une seringue et d'une téterelle faciliterait cette culture. Cependant, la logistique de ces outils était, pour un grand nombre des participantes, laborieuse et insatisfaisante (tableau 2).

À titre d'instrument incorporé aux pratiques de soins favorables à l'allaitement, le tire-lait se retrouvait au centre de plusieurs discours des participantes. En l'occurrence, le tirelait aurait permis, avec la médicalisation des naissances, de dissocier la lactation du phénomène de l'allaitement[37], de rendre facultative la mise au sein dans le désir de donner du lait maternel à son nourrisson ou de quantifier les boires.[38] Une participante de cette étude se positionnait en rupture avec la façon dont était utilisé le tire-lait en milieu hospitalier.

Je n'ai jamais, jamais, jamais, jamais, tiré mon lait pour le (bébé) faire boire. On dirait qu'ils (le personnel hospitalier) m'ont vraiment écœuré. C'est comme s'ils m'avaient coupé le goût. Ils m'ont écœuré en me levant la nuit, en m'obligeant (P6).

Irrémédiablement, le biberon était soit ignoré, mis sous silence ou présenté dans une perspective de peur. Cette peur faisait référence au risque d'un refus ultérieur du sein chez le nourrisson. En ce sens, la neuvième condition pour un succès de l'allaitement maternel, critère de certification de I'IAB, propose d'interdire les tétines aux bébés allaités au sein.[5] Dans cette visée, offrir le biberon ou la suce à son bébé pouvait susciter de la culpabilité chez certaines mères:

C'était que si je donnais la suce, il (le bébé) allait s'habituer et il n'allait plus vouloir faire l'effort de téter. Donc, à partir du moment où moi je lui donne une suce, moi je lui donne un biberon, puis que suite à cela, il ne tète plus, c'est de ma faute (P3).

Cette confusion entre le sein et la tétine s'expliquerait par la différence dans le développement des habiletés motrices orales exigées pour un allaitement comparativement à la succion d'une tétine.[39] Toutefois, pour cette participante, cette peur allait à l'encontre des expériences positives associées à la maternité.

On te fait tellement peur du fait que le bébé ne reprendra pas le sein. S'il (le bébé) a gouté à un biberon. Puis moi là-dessus ça n'a pas été ça. J'ai un $100 \%$ de vécu où mes deux bébés ont repris, $100 \%$ le sein (...) On te fait peur avec la suce, puis avec ci, puis avec ça. (...). C'est ridicule parce qu'on empêche des femmes de vivre des beaux moments, je pense (P6).

Cette mise sous silence du biberon, affirme une autre participante, rendrait ainsi tout échec de l'allaitement exclusif impossible : "Jamais qu'elle (l'infirmière) ne m'a dit : Écoute, si vraiment tu vois que ça ne marche pas, tu peux aller vers des biberons. Jamais! Cela n'a jamais été une possibilité proposée. Jamais, jamais. Jamais " (P9). Pourtant, Jones et Stoppard[40] présentent le transfert d'informations alternatives à l'allaitement, dont l'utilisation du biberon, telle

\begin{tabular}{|l|c|c|c|}
\hline \multicolumn{2}{|c|}{ Tableau 3. Cohabitation des paradigmes de la culture de soin favorable à l'allaitement et de la médicalisation des naissances } \\
\hline & $\begin{array}{c}\text { Culture de soin favorable à } \\
\text { l'allaitement }\end{array}$ & Médicalisation des naissances & $\begin{array}{c}\text { Tensions issues de la cohabitation } \\
\text { des deux paradigmes }\end{array}$ \\
\hline Focalisation & Femme & Sein & Enfant \\
\hline Processus & Allaitement (social) & Lactation (biologique) & Nutrition \\
\hline Approche & Holistique & Technique & Centrée sur l'enfant \\
\hline Principe directeur & Autonomisation & Assujettissement au savoir expert & Assujettissement normatif \\
\hline
\end{tabular}


une pratique favorable à un processus décisionnel éclairé. Cette prise de décision éclairée nécessite par conséquent d'entrevoir et de considérer pleinement les difficultés de l'allaitement maternel exclusif, plutôt que de représenter cette (im)possibilité comme un échec personnel.

Finalement, différentes études critiquent l'approche biomédicale de l'allaitement et proposent que les besoins des mères soient davantage au centre des soins.[41,42,43,10] L'approche centrée sur la personne a été très significative dans le parcours d'une participante. Ses propos exposent les dimensions relationnelles octroyant par le fait même un accompagnement individualisé :

On s'entend-tu que dans le milieu de la santé, tu en fais des affaires en deux heures. Tu en pèses des bébés. Puis elle (l'infirmière) s'est assise et elle m'a écoutée. Puis elle m'a donné des conseils et a vraiment fait une super job (P6).

L'analyse des résultats de cette section présente une tension importante au sein du discours des participantes. Cette tension s'expliquerait notamment par la cohabitation des deux paradigmes : la médicalisation des naissances et de la culture de soins favorable à l'allaitement exclusif[35] (tableau 3). Cette cohabitation aurait pour effet d'induire un sentiment de culpabilisation ou d'incertitude chez plusieurs participantes. Les frontières entre les discours dominants sont fluides et communicatives[25] soumettant une rencontre parfois harmonieuse et parfois discordante.

\section{Discussion}

Au sein des discours provenant des expériences des mères de cette étude, le choix d'une alternative à l'allaitement exclusif se confronte à un ensemble hétérogène de normes sociales et de pratiques institutionnelles, qui se heurtent, ou s'arriment selon une force variable. Selon une compréhension culturelle du soin, ces discours se regroupent principalement sous l'ordre de: la confusion, l'accommodation, l'appréhension, ainsi que la justification.

La confusion. Les résultats proposent une rencontre entre deux mouvements d'influence dans les institutions de santé qui offrent des soins de périnatalité (la médicalisation des naissances et la culture de soin favorable à l'allaitement). La cohabitation de ces deux mouvements pouvait faire vivre de la confusion aux participantes. Les résultats soulèvent une dissonance dans l'anamnèse de l'infirmière qui, dans sa pratique, doit favoriser l'allaitement afin d'être culturellement congruente à la culture de soin et qui cherche à la fois à contrôler certains paramètres médicaux, dont les ingestions du bébé. En effet, les mères qui avaient allaité durant
I'hospitalisation demeuraient perplexes et confuses lorsque les infirmières leur demandaient la quantité de lait ingéré par le bébé. Une étude propose en ce sens que les mères peuvent vivre de la confusion, voire de la colère, devant les conseils contradictoires des professionnels de la santé.[20] Conséquemment, il pourrait être pertinent pour les infirmières de surveiller les différents signes proposés par Ross[44] (nombre de mictions, comportement du bébé après la tétée, quantité et couleurs des selles, état des seins, prise de poids du nourrisson, etc.) plutôt que de questionner les mères sur la quantité de lait donnée chez celles qui font des allaitements avec mise au sein.

L'accommodement. Le discours d'expertise en allaitement qui découle du savoir dominant positionnait certaines mères de cette étude en situation d'apprenantes. Différentes stratégies informelles et persuasives dont les attitudes, l'utilisation d'une posture d'autorité ou encore par le choix des mots permettait de présenter les enjeux de pouvoir entre l'infirmière et la patiente.

Des silences percutants jaillissaient de cette sélection linguistique telle que l'absence de présentations des désavantages de l'allaitement. Ce silence reflète ainsi une rationalité assumée, de la part des acteurs et actrices investis d'un rôle en santé publique visant à souligner les informations favorables à l'allaitement exclusif et minimiser l'importance des informations pouvant nuire à l'atteinte d'un tel objectif. Informer les mères des avantages de l'allaitement tout en mettant sous silence les désavantages de l'allaitement expose une forme de contrôle de l'information qu'opère la santé publique afin de minimiser les comportements jugés risqués pour la santé.[17] Dans cette visée, l'allaitement serait présenté comme un choix sécuritaire et la formule lactée serait socialement présentée tel un comportement risqué pour la santé ou s'écartant du meilleur intérêt du nourrisson.[15,17,29] Pourtant, certains auteurs proposent que la santé publique doive davantage miser sur l'éducation afin de fournir les outils nécessaires pour favoriser des prises de décisions éclairées.[33] De plus, la santé publique devrait aussi différencier les objectifs d'informer et de convaincre les populations, car la persuasion évoque un paternalisme défavorable à l'autonomie des mères, dont les effets néfastes sur leurs expériences d'allaitement et de non-allaitement ont été démontrés par cette étude.[45] En sommes, une approche infirmière centrée sur la personne ainsi qu'une plus grande présentation des alternatives à l'allaitement maternel ainsi que leurs avantages pourrait favoriser l'autonomie des mères.

L'appréhension. Le vécu associé à l'allaitement peut être caractérisé par différentes craintes chez les mères, qui sont 
expliquées par l'attitude autoritaire du personnel soignant et qui peuvent également inciter les mères à s'autocensurer, voire mentir aux infirmières à l'égard du mode d'alimentation choisi. D'abord, cette appréhension dans un contexte de soins va à l'encontre des propriétés attendues du consentement aux soins, qui doit être formulé librement et en connaissance de cause.[46] Ensuite, les connaissances à l'égard des informations portant sur l'allaitement maternel doivent permettre à la patiente d'exercer son pouvoir décisionnel et l'espace relationnel dans lequel évolue cette dernière doit faciliter cette prise de décision. Ainsi, la pondération qu'effectuent les mères vis-à-vis les éléments qu'elles jugent importants ne peut être faite qu'en ayant une connaissance générale de tous les enjeux concernés par une situation, dans un contexte exempt de craintes relié au choix qui sera réalisé. Dans un contexte de soins culturellement congruent à l'allaitement maternel, l'approche de Leininger[24] présente une ouverture au processus décisionnel des mères. Cette ouverture permetà l'infirmière une négociation et parfois même une restructuration du soin culturellement congruent afin de rendre compte des perspectives partagées ou dissonantes de la soignée.[24] Dans cette perspective, il devient possible pour l'infirmière qui adhère pleinement à la culture de soins favorable à l'allaitement d'offrir des soins individualisés qui répondent aux besoins de la parturiente. Or, négocier ou restructurer le soin offert aux femmes qui choisissent une alternative à l'allaitement maternel exclusif permet de tendre vers une congruence culturelle. En contrepartie, maintenir un soin favorable à l'allaitement maternel exclusif chez les mères qui choisissent une alternative à l'allaitement octroie une dissonance de soin et peut contribuer à l'iniquité des relations de pouvoir entre l'infirmière et la parturiente. En conséquence, cette iniquité s'exprime à certains égards par un discours d'appréhension. L'enjeu n'est donc pas de remettre en question l'importance de l'allaitement maternel exclusif, mais d'assurer la mise en œuvre de pratiques flexibles et respectueuses du vécu des mères.

La justification. Dans la littérature scientifique et auprès des participantes de cette étude, les bénéfices de l'allaitement ont été soulignés à grands traits. Actuellement, peu de mères ignorent que l'allaitement est le choix optimal pour la santé des nourrissons.[47] Conséquemment, toutes les participantes de cette étude avaient choisi initialement l'allaitement. Ce choix initial expose l'influence de la norme actuelle qui est favorable à l'allaitement dans leur processus décisionnel. Dans cette visée, cette adhérence à la norme peut octroyer une forte pression chez les mères et Bayard[47] s'interroge sur la moralité de cette norme, qui aurait pour effet d'instaurer une ligne de partage entre les " bonnes " et les " mauvaises " mères. De plus, Knaak[15] suggère qu'un choix alternatif à l'allaitement maternel exclusif soit actuellement perçu comme étant moralement déviant au sein des sociétés contemporaines. La pression sociale d'allaiter son nourrisson a été soulignée par plusieurs mères de cette étude, ce qui illustre bien cette ligne de partage à la fois symbolique et subjective. Dans de telles circonstances, les injonctions morales associées à la maternité auraient un effet marqué sur le choix que font les mères à l'égard du mode d'alimentation du nourrisson. La pression des instances officielles et du discours d'expertise a aussi pour effet de positionner l'allaitement au-delà d'une simple décision individuelle, puisqu'elle implique également et directement le bien-être du nourrisson.[15] Dans la société actuelle, la valorisation du lait maternel et la dévalorisation des formules lactée participeraient au conditionnement des croyances et des décisions au regard du mode d'alimentation du nourrisson choisi.[15]

\section{Avantages et limites de la recherche}

L'originalité de cette recherche s'appuie principalement sur l'apport du cadre conceptuel infirmier de Leininger[24] présenté sous l'angle de la normativité sociale de l'allaitement afin d'en faire une analyse situationnelle.[25] Cette approche théorique a permis d'observer les enjeux culturels de l'allaitement afin de mieux comprendre les particularités du processus de négociation ou de restructuration du soin lorsque des mères choisissent des alternatives. À cet effet, cette étude fait l'état d'expériences de mères qui habitent un pays industrialisé octroyant des enjeux d'allaitements bien différents des pays en émergence. Bien que le modèle théorique de Leininger[24] ait permis d'observer les enjeux culturels de l'allaitement maternel, ce dernier présente certaines limites, la plus évidente étant la considération de la sociologie des genres participant à ce phénomène. Néanmoins, la contribution de l'analyse situationnelle comme méthode de recherche a permis de mettre en lumière la diversité et la complexité de la construction des rapports identitaires au seuil d'une représentation de la maternité véhiculée comme étant naturelle et instinctive. Dans cette visée, le modèle théorique de Leininger[24] ne permettait pas à lui seul de tenir compte des tensions identitaires retrouvées dans les concepts sensibilisants de la maternité intensive[17] ou du sacrifice féminin de la maternité[26] puisqu'il ne propose pas l'analyse directe des discours portant sur l'allaitement, un des principaux vecteurs de cette norme dite biologique de la maternité, maintes fois déconstruite par les théories féministes, spécialement par Badinter.[24]

Les résultats de cette recherche visent un avancement des 
connaissances en sciences infirmières sur un phénomène peu étudié et positionnent l'allaitement tel un enjeu qui s'inscrit dans un contexte global et culturel afin de comprendre le phénomène avec ouverture. Cependant, l'état des connaissances est peu développé sur le sujet ce qui soumet une limite quant à la quantité d'articles scientifiques pertinents et récents. Les résultats de cette étude qualitative détiennent un petit échantillon et ne visent pas la généralisation du phénomène à l'ensemble de la population des mères qui choisissent des alternatives à l'allaitement exclusif. Ces résultats permettent d'apprécier les discours qui réfèrent à des limites normatives et éthiques relatives aux injonctions sociales ciblant l'allaitement. La documentation des discours des participantes de cette étude possède également l'avantage de fournir différentes pistes de réflexion afin de contribuer à l'émergence de pratiques plus sensibles, adaptées et respectueuses du vécu des mères qui font le choix d'une alternative à l'allaitement exclusif. Malgré son efficacité notable, le recrutement en ligne demeurait limité aux personnes présentes sur les réseaux sociaux et qui ont une facilité à lire et s'exprimer sur cette plate-forme. De plus, pour certaines mères, les expériences de naissances dataient de quelques années ce qui peut altérer potentiellement la mémoire de certains souvenirs. Finalement, l'ensemble des recherches recensées ainsi que l'échantillon de l'étude ne présentaient que des couples hétérosexuels. À cet effet, Lee[48] soulève l'importance d'apporter une sensibilité aux communautés LGBTQ+ dans les études portant sur l'allaitement.

\section{References}

1.Binns C, Lee M, Low WY. The Long-Term Public Health Benefits of Breastfeeding. Asia-Pacific. Journal of Public Health 2016; 28(1): 7-14.

2.Colen CG, Ramey D M. Is best truly best? Estimating the effects of breastfeeding on long-terme child health and wellbeing in the United States using sibling comparisons. Social, Science and Medicine 2014; 109: 55-65.

3.Organisation mondiale de la santé. Allaitement maternel exclusif. 2021. Disponible sur http://www.who.int/elena/ titles/exclusive_breastfeeding/fr/. Consulté le 23 avril 2021.

4.UNICEF. Allaitement. 2021. Disponible sur http://www. unicef.org/french/nutrition/index_24824.html. Consulté le 23 avril 2021.

5.Ministère de la Santé et Services sociaux du Québec. Initiative des amis des bébés. 2021. Disponible sur http:// www.msss.gouv.qc.ca/professionnels/amis-des-bebes/
Consulté le 23 avril 2021.

6. Ordre des infirmières et infirmiers du Québec. Standards de pratique de l'infirmière : Soins de proximité en périnatalité. 2015. Disponible sur http://www.oiiq.org/sites/default/ files/4443-perinatalite-web.pdf. Consulté le 2 novembre 2020.

7.Ordre des Sages-Femmes du Québec. Philosophie et normes de pratique. 2020. Disponible sur : https://www.osfq.org/ quest-ce-quune-sage-femme/philosophie-et-normes-depratiques/ Consulté le 2 novembre 2020.

8.Goberna-Tricas J, Banus-Gimenez R, Palacio-Tauste A., Linares-Sancho S. Satisfaction with pregnancy and birth services: The quality of maternity care service and experienced by women. Midwifery 2011; 27: 231-237.

9.Murphy E. "Breast is best": infant feeding decisions and maternal deviance. Sociology of Health and IIIness. 1999; 21(2): 187-208.

10.Fallon V, Komninou S, Bennett K M, Halford. J C G, Harrold J A. The emotional and practical experiences of formula-feeding mothers. Maternal and Child Nutrition. 2017; 13(4): 1-14.

11. Hunt L, Thomson G. Pressure and judgement within a dichotomous landscape on infant feeding: a grounded theory study to explore why breastfeeding women do not accesd to peer support provision. Maternal and Child Nutrition. 2017; 13(2): 1-13.

12.Hvatum I, Glavin K. Mothers' experience of not breastfeeding in a breastfeeding culture. Journal of Clinical Nursing. 2016; 26(19-20): 3144-3155.

13. Wirihana L A, Barnard A. Women's perceptions of their healthcare experience when they choose not to breastfeed. Women and Birth. 2011; 25(3): 135-141.

14.Chabrol H, Walburg V, Teisssedre F, Armitage J, Santrisse K. Influence of mother's perceptions on the choice to breastfeed or bottle-feed: perceptions and feeding choice. Journal of Reproductive and Infant. 2004; 22(3): 189-198.

15.Knaak S J. Contextualising risk, constructing choice: Breastfeeding and good mothering in risk society. Health, Risk \& Society. 2010; 12(4): 345-355.

16.Labbok M. Exploration of guilt among mothers who do not breastfeed: the physician's role. Journal of Human Lactation. 2008; 24(1): 80-84.

17.Lee E. Living with risk in the era of 'intensive motherhood': Maternal identity and infant feeding. Health, Risk and Society. 2008; 10(5): 467-477. 
18. Mander R. Baby friendly-motner friendly? Policy issues in breastfeeding promotion. Midirs Midwifery Digest. 2008; 18(1): 104-108.

19.Nihlén Fahlquist J. Experience of non-breastfeeding mothers. Norms and ethically responsible risk communication. Nursing Ethics 2016; 23(2): 231-241.

20.Redshaw M, Henderson J. Learning the Hard Way: Expectations and Experiences of Infant Feeding Support. Birth Issues in Perinatal Care. 2012; 39(1): 21-29.

21.Lakshman R, Ogilvie D, Ong K K. Mothers' experiences of bottle-feeding: a systematic review of qualitative and quantitative studies. Arch. Dis Child. 2009; 94(8) : 596-601.

22.Chumova, M. Le guide pratique mieux vivre avec notre enfant : témoin des discours et représentations autour de l'allaitement. In : Bayard C, ChouinardC (eds). La promotion de l'allaitement au Québec. Montréal: les Éditions remueménage, 2014; 45-65.

23.Gostin L O. Public health law: power, duty, restraint. (2e ed). Los Angeles: University of California Press, 2008.

24.Leininger M. Culture care theory: a major contribution to advance transcultural nursing knowledge and practice. Journal of Transcultural Nursing. 2002; 13(3): 189-192.

25.Clarke A, Friese C, Washburn R. Situational Analysis. Grounded theory after the interpretative turn. California : Éditions Sage, 2018.

26.Badinter E. Le conflit, la femme et la mère. Paris: Flammarion, 2010.

27.McBride-Henry K. The influence of the "They": An interpretation of breastfeeding culture in New Zealand. Qualitative Health Research. 2010; 20(6): 768-777.

28.Spencer R L, Greatrex-White S, Fraser D M. 'I thought it would keep them all quiet'. Women's experiences of breastfeeding as illusions of compliance: an interpretive phenomenological study. Journal of Advanced Nursing. 2015; 71(5): 1076-1086.

29.Striley K M, Field-Springer K. The bad mother police: theorizing risk orders in the discourses of infant feeding practices. Health Communication. 2014; 29(6): 552-562.

30.Thomson G, Ebisch-Burton K, Flacking R. Shame if you do - shame if you don't: women's experiences of infant feeding. Maternal and Child Nutrition. 2015; 11(1): 33-46.

31.Lee E. Health, morality, and infant feeding: British mothers' experiences of formula milk use in the early weeks. Sociology of Health and IIIness. 2007; 29(7): 1075-1090.

32.Thomson G, Dykes F. Women's Sense of Coherence related to their infant feeding experiences. Maternal and Child Nutrition. 2011; 7(2): 160-174.

33.Wolf J. Is breast really best? Risk and total motherhood in the National Breastfeeding. Awareness Campaign. Journal of health Politics, Policy and Law 2007; 32(4): 59.

34.Eden A R. New professions and old practices. Lactation consulting and the medicalization of breastfeeding. In Smith $\mathrm{P} \mathrm{H}$, Hausman B L, Labbok M. (eds), Beyond health, beyond choice: breastfeeding constraints and realities. New Jersey: Rutgers University Press, 2012, 98-109.

35.Rivard A. Histoire de l'accouchement dans un Québec moderne. Montréal: les Éditions remue-ménage, 2014.

36.Sheehan A, Schmied V, Barclay I. Women's experiences of infant feeding support in the first 6 weeks post-birth. Maternal and Child Nutrition. 2009; 5(2): 138-150.

37.McCarter-Spaulding D. Is breastfeeding fair? Tensions in feminist perspectives on breastfeeding and the family. Journal of Human Lactation. 2008; 24(2): 206-212.

38.Johns H M, Amir L H, McLachlan H L, Forster D A. Brest pump use among mothers of healthy term infants in Melbourne Australia: A prospective cohort study. Midwifery. 2016; 33: 8289.

39.Lowdermilk L D, Perry E S, Cashion K. Périnatalité. Montréal: Chenelière education, 2018.

40.Jones S R, Stoppard M. Baby Friendly Hospital: are we failing mother who formula feed their babies? Journal of family health care. 2011; 21(1): 12-14.

41.Battersby S. The role of the midwife in breastfeeding: Dichotomies and dissonance. British Journal of Midwifery. 2014; 22(8): 551-556.

42.Benoit B, Goldberg L, Campbell-Yeo M. Infant feeding and maternal guilt: The application of a feminist phenomenological framework to guide clinician practices in breast feeding promotion. Midwifery. 2016; 34: 58-65.

43.Carroll M, Gallagher L, Clarke M, Lillar S, Begley C. Artificial milk-feeding women's views of their feeding choice in Ireland. Midwifery. 2015; 31 : 640-646.

44.Ross L. Guide de santé postnatale 9 mois plus tard. 2003. Disponible sur http://collections.banq.qc.ca/ark:/52327/ bs57392. Consulté le 2 novembre 2020.

45.Massé R. Éthique et santé publique. Enjeux, valeurs et 
normativité. (6e ed). Québec: Les presses de I'Université Laval, 2014.

46.Code civil du Québec. RLRQ c CCQ-1991. 1991. Disponible sur http://legisquebec.gouv.qc.ca/fr/ShowDoc/cs/CCQ-1991 Consulté le 2 novembre 2020.

47. Bayard C. Introduction. In Bayard C, Chouinard C. (eds), La promotion de l'allaitement au Québec. Montréal: les éditions remue-ménage, 2014, 17-22.

48.Lee R. Queering lactation: contributions of queer theory to lactation support for LGBTQIA2S+ individuals and families. Journal of Human Lactation. 2019; 35(2): 233-238.

49.Pepin J, Ducharme F,Kérouac S. La pensée infirmière (4e ed). Montréal : Chenelière Éducation, 2017.

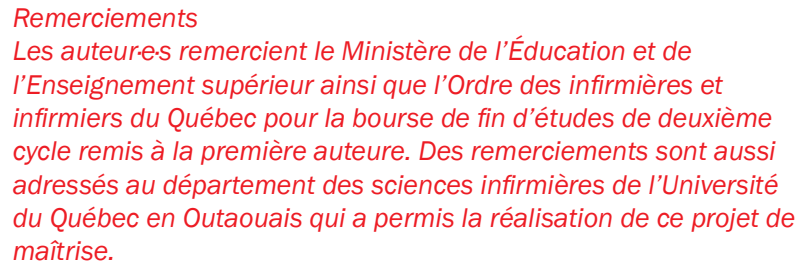

Pour contacter les auteur-e-s:

Sandrine Vallée-Ouimet, Inf. M.Sc.

Université du Québec en Outaouais

5, rue St-Joseph

Saint-Jérôme, Québec, J7Z OB7

Canada

Courriel: Sandrinev.ouimet@hotmail.com

Benoit, Monique, Ph,D.

Université du Québec en Outaouais

Département des sciences infirmières

Pierre Pariseau-Legault, Inf., Ph.D., LLM.

Université du Québec en Outaouais

Département des sciences infirmières 